\title{
Morning plasma cortisol as a cardiovascular risk factor: findings from prospective cohort and Mendelian randomization studies
}

\author{
Andrew A Crawford ${ }^{1,2,3}$, Stefan Soderberg4, Clemens Kirschbaum5, Lee Murphy6, Mats Eliasson', Shah Ebrahim ${ }^{8}$, \\ George Davey Smith ${ }^{2,3}$, Tommy Olsson ${ }^{4}$, Naveed Sattar9, Debbie A Lawlor ${ }^{2,3}$, Nicolas J Timpson ${ }^{2,3}$, \\ Rebecca M Reynolds ${ }^{1}$ and Brian R Walker ${ }^{1,10}$
}

${ }^{1}$ BHF Centre for Cardiovascular Science, Queen's Medical Research Institute, University of Edinburgh, Edinburgh, UK, ${ }^{2} \mathrm{MRC}$ Integrative Epidemiology Unit at the University of Bristol, Bristol, UK, ${ }^{3}$ Population Health Sciences, Bristol Medical School, University of Bristol, Bristol, UK, ${ }^{4}$ Department of Public Health and Clinical Medicine, Umeå University, Umeå, Sweden, ${ }^{5}$ Department of Psychology, Dresden University of Technology, Dresden, Germany, ${ }^{6}$ Edinburgh Clinical Research Facility, University of Edinburgh, Edinburgh, UK, ${ }^{7}$ Department of Public Health and Clinical Medicine, Sunderby Research Unit, Umeå University, Umeå, Sweden, ${ }^{8}$ Department of Epidemiology \& Population Health, London School of Hygiene \& Tropical Medicine, London, UK, ${ }^{9}$ Division of Cardiovascular and Medical Sciences, University of Glasgow, Glasgow, UK, and ${ }^{10}$ Institute of Genetic Medicine, Newcastle University, Newcastle upon Tyne, UK

Correspondence should be addressed to B R Walker

Email

Brian.Walker@ncl.ac.uk

\begin{abstract}
Objective: The identification of new causal risk factors has the potential to improve cardiovascular disease (CVD) risk prediction and the development of new treatments to reduce CVD deaths. In the general population, we sought to determine whether cortisol is a causal risk factor for CVD and coronary heart disease (CHD).

Design and methods: Three approaches were adopted to investigate the association between cortisol and CVD/CHD. First, we used multivariable regression in two prospective nested case-control studies (total 798 participants, 313 incident CVD/CHD with complete data). Second, a random-effects meta-analysis of these data and previously published prospective associations was performed (total 6680 controls, 696 incident CVD/CHD). Finally, one- and two-sample Mendelian randomization analyses were performed (122,737 CHD cases, 547,261 controls for two-sample analyses). Results: In the two prospective nested case-control studies, logistic regression adjusting for sex, age, BMI, smoking and time of sampling, demonstrated a positive association between morning plasma cortisol and incident CVD (OR: 1.28 per 1 SD higher cortisol, $95 \% \mathrm{Cl}: 1.06-1.54)$. In the meta-analysis of prospective studies, the equivalent result was OR: 1.18, 95\% Cl: 1.06-1.31. Results from the two-sample Mendelian randomization were consistent with these positive associations: OR: $1.06,95 \% \mathrm{Cl}: 0.98-1.15$.

Conclusions: All three approaches demonstrated a positive association between morning plasma cortisol and incident CVD. Together, these findings suggest that elevated morning cortisol is a causal risk factor for CVD. The current data suggest strategies targeted at lowering cortisol action should be evaluated for their effects on CVD.
\end{abstract}

\section{Introduction}

Despite lifestyle improvements and the successful targeting of cardiovascular disease (CVD) risk factors, such as hypercholesterolaemia (1) and hypertension (2), CVD is a leading cause of death in high-income countries and increasingly so in low- and middleincome countries (3). The identification of new causal risk factors, such as elevated morning plasma cortisol, has the potential to improve CVD risk prediction https://eje.bioscientifica.com https://doi.org/10.1530/EJE-19-0161 Printed in Great Britain
This work is licensed under a Creative Commons Attribution-NonCommercial-NoDerivatives 4.0 International License.

ed from Bioscientifica.com at 04/26/2023 01:45: $02 \mathrm{PM}$ 
and the development of new treatments to reduce CVD deaths.

Cortisol is a glucocorticoid produced by the adrenal glands, which is responsible for cardiovascular and metabolic adaptations during stress. Plasma cortisol has a circadian rhythm with a peak on waking and then declining throughout the day. Inappropriately sustained cortisol production in patients with tumours causing Cushing's syndrome results in abdominal obesity, hyperglycaemia, hypertension and dyslipidaemia and is associated with a four-fold increase in mortality rate, predominantly due to accelerated atherosclerosis and increased CVD $(4,5)$. A similar higher rate of CVD has been documented in patients prescribed exogenous glucocorticoid therapy at supra-physiological doses (6).

Several population-based cross-sectional studies have reported morning plasma levels of cortisol to be positively associated with plasma glucose, blood pressure and other cardiovascular risk factors $(7,8,9,10)$. Associations have also been found between cortisol and markers of subclinical atherosclerosis including carotid plaques and coronary artery calcification (11). Evidence of an association between circulating cortisol levels and CVD events is, however, less conclusive, with studies to date mostly being cross-sectional (12). Three prospective cohort studies have reported a positive association between morning plasma cortisol and CVD, but effects were imprecisely estimated due to the small study sizes (number of incident CVD cases between 63 and 320) (13, $14,15)$. Larger prospective studies, and the use of genetic predictors of morning plasma cortisol as instrumental variables in Mendelian randomization analyses (16), are required to address whether cortisol is a causal risk factor for CVD.

A recent genome-wide association meta-analysis (GWAMA) study identified independent variants in one locus on chromosome 14 which together explained $0.5 \%$ of the variance in morning plasma cortisol in European populations (17). These findings provide instrumental variables for a Mendelian randomization analysis of the effect of morning plasma cortisol on CVD (16).

We hypothesised that elevated morning plasma cortisol is a causative risk factor for incident CVD and tested this in multiple prospective studies and using Mendelian randomization.

\section{Methods}

\section{New prospective cohort studies of plasma cortisol and CVD}

The Northern Sweden VIP, MONICA and MSP study

A prospective case-control study of fatal and non-fatal myocardial infarction (MI) and stroke was nested within the combined data from three studies conducted in the same population (Northern Sweden) using identical study protocols (the Västerbotten Intervention Program (VIP) (18), the Northern Sweden MONItoring Of trends and Determinants in CArdiovascular Disease (MONICA) survey and the Mammary Screening Program (MSP); details are provided in Supplementary material, see section on supplementary data given at the end of this article) $(19,20)$. Incident cases were selected after removal of all prevalent cases, and two controls for every case were matched to cases by sex, age (median difference in age 0.33 years), study and date of baseline assessment (median difference in baseline assessment date 79 days). The study was approved by the Research Ethics Committee of Umeå University and informed consent was obtained from all participants.

Cortisol was measured by immunoassay on plasma samples obtained at the baseline assessment after at least $4 \mathrm{~h}$ of fasting and stored at $-80^{\circ} \mathrm{C}(21)$. Genotyping of three SNPs from the GWAMA which together contributed most to morning plasma cortisol (rs12589136, rs2749527, rs11621961) (17) was performed on the OpenArray platform.

\section{British Women's Heart and Health Study (BWHHS)}

BWHHS is a prospective cohort study of 4286 women who were randomly selected from 23 British towns between 1999 and 2001 and who were aged 60-79 years at baseline; details are provided in Supplementary material (22). We used participants from a prospective case-control study of coronary heart disease (CHD) nested within this cohort, as previously described (23). Incident cases of CHD were selected, and two controls were randomly selected, within 5 -year age groups of the cases, from women without CHD at the baseline assessment and who had been followed up over the same time as the cases without a CHD event.

Serum cortisol levels were determined by radioimmunoassay (MP Biomedicals, UK; intra-assay CV 
5.1-7.0\%, inter-assay CV 6.0-7.9\%) on blood samples obtained at the baseline examination after a minimum 6-h fast between 08:00 $\mathrm{h}$ and 17:00 h. The BWHHS has Local- and Multi-Centre Research Ethics Committee (LREC and MREC) approvals and all participants provided written informed consent.

\section{Statistical analysis}

In the VIP, MONICA and MSP cohort, conditional logistic regression models, adjusting for matching variables (age and baseline assessment date), smoking, body mass index (BMI) and time of sampling (continuous variable as number of minutes since the earliest sampling time) were used to investigate the effect of a 1 s.D. increase in morning plasma cortisol on CVD. Sex and study did not vary between matched cases and controls and so were not included in the model. The controls in BWHHS were matched to cases within 5-year age bands meaning control for the matching factor can be obtained, with no loss of validity and possible increase in precision, using (unconditional) logistic regression as there are no problems of sparse data (24). In BWHHS, logistic regression models, adjusting for the matching variable (age), smoking, BMI and time of sampling were used to investigate the effect of a 1 s.D. increase in morning plasma cortisol on CHD.

The main analysis used samples collected before 11:00 $\mathrm{h}$, adjusted for time of sampling, as used for the GWAMA which identified genetic variants associated with morning plasma cortisol (17). The impact of missing data was investigated using multiple imputation by chained equations (25); details of this procedure and all additional analyses are in the Supplementary material. Analyses were performed using Stata v.14 (26). We report exact two-sided $P$ values throughout.

\section{Meta-analysis of prospective studies}

The multivariable regression meta-analysis of prospective studies combined data from the unpublished analyses of VIP, MONICA and MSP (fatal and non-fatal CVD) and BWHHS (fatal and non-fatal CHD) described earlier, as well as published data for morning plasma cortisol from the Caerphilly Study (fatal and non-fatal ischaemic heart disease (IHD)) (13) and the Vietnam Experience Study (fatal CVD) (15). Details of these cohorts are available in the Supplementary material. We were not able to include a third published prospective study (14) because the necessary data were not available.

\section{Statistical analysis}

A random-effects (DerSimonian-Laird) method was used to pool the maximally confounder adjusted (for each study) association of 1 s.D. higher morning plasma cortisol with CVD/CHD. A random-effect method was used as we were aware of clinical/study heterogeneity (i.e. different confounder adjustment, outcome definitions and genders/geographical locations), and statistical evidence of heterogeneity between studies was assessed with chisquared goodness of fit and the $I^{2}$ statistic, together with its $95 \%$ CI (27). Sensitivity analyses are described in the Supplementary material.

\section{One- and two-sample Mendelian randomization analyses}

Mendelian randomization analyses use genetic variants as a proxy for environmental exposures. As genetic variants are assigned at conception and tend not to be associated with other behavioural, social or physiological factors, these analyses are less likely to be affected by reverse causation or confounding than conventional observational studies (16). The genetic instrument for morning plasma cortisol comprised three SNPs (rs12589136, rs2749527, rs11621961) in low linkage disequilibrium $\left(r^{2}<0.3\right)$, each associated independently with morning plasma cortisol in the CORtisol NETwork (CORNET) GWAMA $(n=12,597)$ (17). Biochemical investigation of these SNPs have also shown them to be associated with variation in total cortisol-binding activity in plasma (17). The three SNPs explain approximately $0.5 \%$ variation in morning plasma cortisol levels and are located on chromosome 14 at a locus containing the SERPINA6 gene. The SERPINA6 gene codes for corticosteroid-binding globulin (CBG) which binds to cortisol in the blood. To assess the potential of horizontal pleiotropy, where a genetic variant influences multiple outcomes through independent pathways, we investigated the association of these SNPs with confounders included in the multivariable prospective analysis in the VIP, MONICA and MSP cohort and with cardiovascular risk factors in publicly available GWAS consortia.

\section{One-sample Mendelian randomization analysis in VIP, MONICA and MSP cohort}

The causal estimate was derived using the two-stage method comprising a first-stage regression of the exposure on the SNP and a second-stage regression of the outcome 
on the fitted values of the exposure from the first stage. Further details are available in the Supplementary material. This provides the log OR for CVD per 1 s.D. increase in morning plasma cortisol due to each SNP. The effects of each SNP were combined in a random-effects meta-analysis.

\section{Two-sample Mendelian randomization analysis}

A two-sample Mendelian randomization approach (28), using publicly available data, was used to estimate the causal effect of morning plasma cortisol on CHD. We investigated the association between the genetic instrument for morning plasma cortisol and risk of CHD in a meta-analysis of 34,541 CHD cases and 261,984 controls of UK Biobank and 88,192 cases and 162,544 controls from CARDIoGRAMplusC4D (29). In the main analyses the causal effect of morning plasma cortisol on CHD was estimated for each SNP individually using the Wald ratio method (30) and then combined in a random-effects meta-analysis. Additional analyses described in the Supplementary material were performed to investigate the robustness of this causal estimate and any potential pleiotropic effects. The summary GWAS data for morning plasma cortisol and CHD can be downloaded from https://datashare.is.ed. ac.uk/handle/10283/2787 and https://data.mendeley. com/datasets/gbbsrpx6bs/1 respectively.

\section{Results}

\section{New prospective cohort studies of plasma cortisol and CVD}

The Northern Sweden VIP, MONICA and MSP study

There were 905 cases and 1717 matched controls (93 cases with one control, 812 cases with two controls) included in the analysis. Of the 905 cases of CVD, there were 536 cases of MI and 369 cases of stroke, including fatal and non-fatal events. Median time to CVD event was 3 years 9 months. Those with CHD were more likely to have hypertension and higher BMI, total cholesterol, triglycerides and fasting glucose and to smoke tobacco (Table 1). Owing to the high proportion with missing data (Supplementary Table 4), analyses were performed for all participants, those with complete data, and in a dataset with imputation of missing data. Time of blood sampling was not recorded in $52 \%$ of participants, who were more likely to be male, not fasted, and have lower BMI, leptin and fasting glucose levels (Supplementary Table 5). The variables used in the imputation models are presented in Supplementary Table 6 and distributions of imputed variables in Supplementary Table 7.

There was no association between plasma cortisol and CVD incidence in the unadjusted model of all participants (Table 2). However, there was a positive association between morning plasma cortisol and CVD when including only participants with cortisol assessed

Table 1 Characteristics of cases and controls in the combined VIP, MONICA and MSP cohort and BWHHS cohort. Means and standard deviations are presented for continuous variables. The number and percentage of individuals are presented for binary variables. Hypertensive was generated as blood pressure at examination $\geq 160 / 90 \mathrm{mmHg}$ and/or taking antihypertensive medication. The $P$ value from VIP, MONICA and MSP is from a conditional logistic regression model accounting for the matched nature of the data.

\begin{tabular}{|c|c|c|c|c|c|c|}
\hline & \multicolumn{3}{|c|}{ VIP, MONICA and MSP } & \multicolumn{3}{|c|}{ BWHнS } \\
\hline & Cases of ICHD $(n=905)$ & Controls $(n=1717)$ & $P$ & Cases of ICHD $(n=169)$ & Controls $(n=327)$ & $P$ \\
\hline Cortisol, nmol/L (s.D.) & 524 (193) & 527 (183) & 0.85 & $526(222)$ & $530(207)$ & 0.58 \\
\hline Male, $n(\%)$ & $655(72)$ & $1236(72)$ & - & $0(0)$ & $0(0)$ & - \\
\hline Age, years (s.D.) & $54.5(7.8)$ & $54.3(7.9)$ & 0.11 & $70.4(5.5)$ & $70.1(5.3)$ & 0.47 \\
\hline BMI, kg/m² (s.D.) & $27.0(3.9)$ & $25.9(3.6)$ & $<0.0001$ & $28.1(4.9)$ & $27.4(4.5)$ & 0.14 \\
\hline FGL, mmol/L (s.D.) & $5.63(1.70)$ & $5.36(1.12)$ & $<0.0001$ & $6.29(2.17)$ & $6.20(1.99)$ & 0.65 \\
\hline Total cholesterol, mmol/L (s.D.) & $6.54(1.30)$ & $6.17(1.25)$ & $<0.0001$ & $6.76(1.41)$ & $6.63(1.13)$ & 0.27 \\
\hline HDL cholesterol, mmol/L (s.D.) & $1.25(0.98)$ & $1.37(0.92)$ & 0.18 & $1.53(0.42)$ & $1.68(0.47)$ & 0.0003 \\
\hline Triglycerides, mmol/L (s.D.) & $1.89(1.27)$ & $1.54(0.87)$ & $<0.0001$ & $2.10(1.02)$ & $1.87(1.00)$ & 0.005 \\
\hline SBP, mmHg (S.D.) & $142(19)$ & $135(17)$ & $<0.0001$ & $153(22)$ & $148(27)$ & 0.05 \\
\hline DBP, mmHg (s.D.) & $89(10)$ & $85(9)$ & $<0.0001$ & $81(11)$ & $80(12)$ & 0.52 \\
\hline Hypertensive, $n(\%)$ & $574(72)$ & $752(50)$ & $<0.0001$ & $136(80)$ & $232(71)$ & 0.02 \\
\hline Ever smoked, $n(\%)$ & $282(33)$ & $337(20)$ & $<0.0001$ & $90(53)$ & $138(42)$ & 0.02 \\
\hline
\end{tabular}


Table 2 Associations of plasma cortisol with CVD in the combined VIP, MONICA and MSP cohort and BWHHS cohort. All models included the matching variables of age; for VIP, MONICA and MSP, models also included the matching variables of sex, study and baseline assessment date. Adjusted models also included BMI, smoking and time of sampling in the regression models. Analyses were repeated restricted to individuals with blood sampling time between $0700 \mathrm{~h}$ and $1100 \mathrm{~h}$.

\begin{tabular}{|c|c|c|c|c|c|c|c|c|}
\hline & \multicolumn{4}{|c|}{ VIP, MONICA and MSP } & \multicolumn{4}{|c|}{ BWHHS } \\
\hline & Cases & Controls & OR $(95 \% \mathrm{Cl})$ & $P$ & Cases & Controls & OR $(95 \% \mathrm{Cl})$ & $P$ \\
\hline Unadjusted & 905 & 1717 & $1.00(0.91-1.09)$ & 0.91 & 169 & 327 & $0.95(0.78-1.14)$ & 0.55 \\
\hline $\begin{array}{l}\text { Adjusted } \\
0700 \mathrm{~h}-1100 \mathrm{~h}\end{array}$ & 323 & 492 & $1.19(1.01-1.41)$ & 0.04 & 154 & 313 & $0.99(0.80-1.22)$ & 0.92 \\
\hline Unadjusted & 297 & 445 & $1.17(0.99-1.39)$ & 0.07 & 45 & 91 & $1.22(0.81-1.85)$ & 0.34 \\
\hline Adjusted & 268 & 396 & $1.31(1.07-1.60)$ & 0.009 & 45 & 89 & $1.13(0.72-1.76)$ & 0.59 \\
\hline
\end{tabular}

$\mathrm{Cl}$, confidence intervals; $\mathrm{OR}$, odds ratio; $P, P$ value.

between $0700 \mathrm{~h}$ and $1100 \mathrm{~h}$ (cases=297, controls=445), which was strengthened when adjusting for potential confounders $($ cases $=268$, controls $=396$ ) $($ Table 2). When the analysis was restricted to participants with complete data, there was evidence of a positive association in all models (Supplementary Table 1). The effect that each confounder has on the effect estimate can be seen in the one-at-a-time analysis with BMI having the largest effect in the observed (Supplementary Table 2) and imputed (Supplementary Table 3) data. The estimate obtained from the imputed data supported an association between higher morning plasma cortisol and CVD, but with an attenuated estimate in the adjusted model (OR 1.12, 95\% CI 0.99-1.26) (Supplementary Table 8).

When the adjusted analysis was stratified by outcome (MI or stroke) the association between morning plasma cortisol and MI appeared to be stronger (OR 1.85, 95\% CI 1.28-2.68) than with stroke (OR 1.08, 95\% CI $0.84-$ 1.39); $P=0.01$ for difference between the two estimates. There was no strong statistical evidence to suggest the association differed by sex $(P=0.36)$.

\section{British Women's Heart and Health Study (BWHHS)}

There were 169 cases (111 non-fatal and 58 fatal) and 363 controls included in the analysis of the BWHHS nested case-control study. Those with CHD had higher triglyceride levels and systolic blood pressure and lower HDL cholesterol levels; they were more likely to have hypertension and to have ever smoked (Table 1). The pattern of associations was similar to that in the VIP, MONICA and MSP studies, with little evidence of an association in unadjusted analyses of the whole study population and a positive association emerging as analyses were restricted to those with a sample taken between $0700 \mathrm{~h}$ and $1100 \mathrm{~h}$ and after adjustment for confounders (Table 2). Estimates in BWHHS were less precise than in
VIP, MONICA and MSP with 95\% confidence intervals including the null value.

\section{Meta-analysis of prospective studies}

By combining data from the previously published analyses of the Caerphilly Study and Vietnam Experience Study with data from the VIP, MONICA and MSP cohorts and BWHHS cohort, a total of 7376 individuals (696 CVD events (fatal and non-fatal), 6680 controls) were included. The estimate from the Vietnam Experience Study was from an unadjusted regression model as that was all that was available. The other estimates were from models adjusting for potential confounders (Supplementary Table 9). A 1 s.D. higher cortisol was associated with 18\% higher odds of experiencing a CVD event (OR: 1.18, 95\% CI: 1.06-1.31) (Fig. 1). There was no strong evidence of heterogeneity $\left(\chi_{[3]}^{2}=3.14, P=0.37, I^{2}=5 \% \quad(95 \%\right.$ CI: $0-86 \%)$, and the positive association remained in a leaveone-out analysis (Supplementary Table 10). Though power for these analyses is low, we found no strong evidence of association between study effect size estimate and mean participant age $(P=0.64)$ or percentage male $(P=0.93)$.

Visual inspection of a funnel plot of the effect size $(\operatorname{lnOR})$ against the standard error of the effect size $(\ln O R)$ suggested asymmetry (Supplementary Fig. 1), indicating that some small studies that were consistent with a small or null association between cortisol and risk of CVD may be missing. Egger's test did not provide evidence of small study bias $(P=0.32)$, although this test has low statistical power given the low number of studies included.

\section{One- and two-sample Mendelian randomization analyses}

In the VIP, MONICA and MSP cohort, there was no strong evidence of an association between the SNPs 


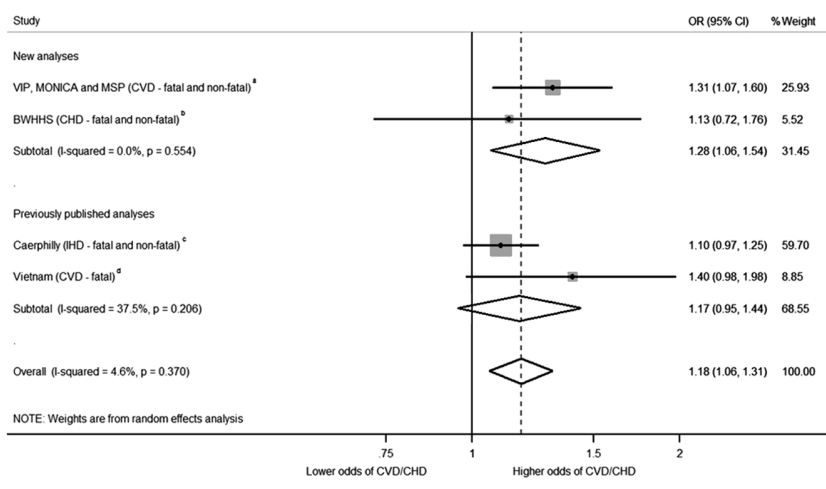

\section{Figure 1}

Meta-analysis of prospective multivariable regression association of morning plasma cortisol with cardiovascular disease. Meta-analysis provides evidence that a 1 S.D. increase of morning plasma cortisol is associated with $18 \%$ higher risk of later cardiovascular disease (odds ratio (OR) 1.18 95\% Cl 1.06-1.31, $P=0.002)$. The VIP, MONICA and MSP study consisted of 268 cases and 396 controls; the BWHHS consisted of 45 cases and 89 controls; the Caerphilly Study consisted of 320 cases and 2003 controls; the Vietnam study consisted of 63 cases and 4192 controls. The number of controls in the Caerphilly and Vietnam Experience studies was assumed to be all individuals who did not experience a CVD event or CVD death. An assumption was made that the standard deviation of cortisol in the Vietnam Experience Study was the same as the VIP, MONICA and MSP study; this allowed inclusion of the Vietnam Experience study. Bars represent individual study 95\% confidence intervals, with a central block proportional to study size. The summary diamond represents the pooled effect size estimate and $95 \% \mathrm{Cl}$. Weights are from randomeffects analysis. ${ }^{a}$ Regression model included age, survey date, cohort, smoking, BMI and sampling time. ${ }^{b}$ Regression model included age, smoking, BMI and sampling time. 'Regression model including age, smoking status, sampling time, adult social class, alcohol consumption, height, FEV1/height ${ }^{2}$,

fibrinogen (log transformed), white cell count (log

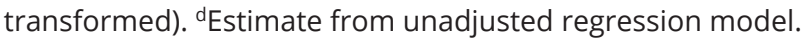

comprising the instrumental variable with any of the matching variables (age, sex, survey date, cohort) or potential confounders (BMI, sampling time, smoking) either individually or when combined in a fixed-effects or random-effects meta-analysis (Supplementary Table 11). In publicly available GWAS consortia the instrumental variable was positively associated with type 2 diabetes and negatively associated with being overweight, LDL cholesterol and cigarettes smoked per day (Supplementary Table 12). The Mendelian randomization analysis in

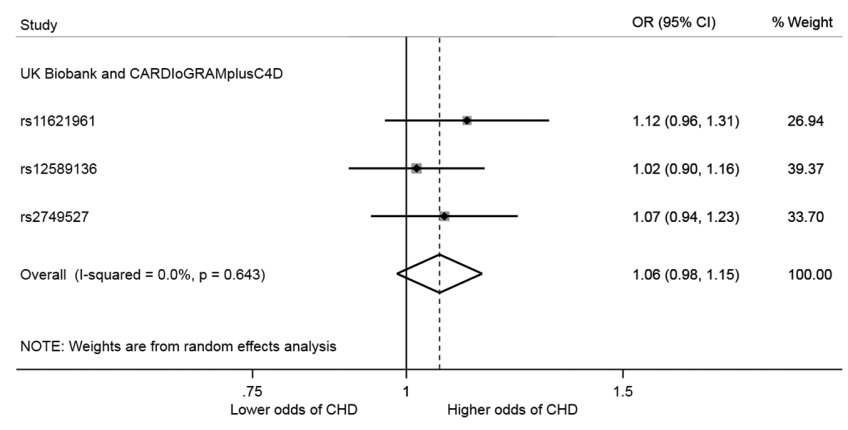

\section{Figure 2}

Effect estimate of genetically elevated morning plasma cortisol on risk of CHD. The forest plot shows odds ratios and $95 \%$ confidence intervals for CHD per standard deviation increase in morning plasma cortisol. The overall genetic effect was estimated using random-effects meta-analysis of Wald ratio estimates. The genetic effect was estimated using data from the meta-analysis of 34,541 CHD cases and 261,984 controls from UK Biobank and 88,192 cases and 162,544 controls from CARDIoGRAMplusC4D (29).

the VIP, MONICA and MSP cohort suggested a positive effect of morning plasma cortisol on CVD risk (OR: 1.33, 95\% CI: 0.26-6.73), but this had a wide 95\% confidence interval given the small sample size.

Using publicly available data, two-sample Mendelian randomization analysis provided evidence that each SD increase in morning plasma cortisol increased CHD: OR: 1.06, 95\% CI: 0.98-1.15 (Fig. 2). There was no strong statistical evidence of heterogeneity between the instrumental variable effect estimate for each SNP (Q statistic $=0.88, P=0.64)$. Estimates obtained from the additional Mendelian randomization analyses, including multivariable Mendelian randomization, lacked precision but consistent with the positive association obtained in the main analyses (Supplementary Tables 13 and 14).

\section{Discussion}

Previous prospective studies have reported positive associations between morning plasma cortisol and later CVD risk but a lack of precision of the effect estimate cast doubt on the importance of cortisol $(13,14,15)$. Moreover, these reports focused on associations with CVD of the ratio of cortisol to testosterone (13), estradiol (14) and DHEAS (15) which are of questionable biological relevance. Here, we focused on morning plasma cortisol and were able to obtain precise estimates of its association with incident CVD/CHD by undertaking analyses in two new 
(previously unpublished) prospective nested case-control studies and pooling those results with existing published prospective studies in a random-effects meta-analysis. Following adjustment for key confounders (age, sex, BMI, smoking) where possible we found that a 1 s.D. higher morning plasma cortisol was associated with 18\% higher odds of incident CVD/CHD. Furthermore, evidence from the Mendelian randomization analyses was consistent with these multivariable results, supporting a positive causal effect of morning cortisol levels on CVD/CHD. Given the key sources of biases in these two approaches are different - residual confounding in the multivariable regression analyses and horizontal pleiotropy, where a genetic variant influences multiple outcomes through independent pathways, in the Mendelian randomization analyses - the consistent finding with each strengthens the conclusion that there is a causal effect (31). Thus, our findings support the development of interventions that effectively lower cortisol as a means of reducing risk of CVD (32).

Plasma cortisol has a circadian rhythm with a peak on waking. In earlier studies, we found that several metabolic cardiovascular risk factors, such as blood glucose and blood pressure, were more closely associated with morning plasma cortisol than with other measurements such as 24-h urinary cortisol metabolites (33), and that these associations are strengthened by adjusting for the time of sampling, at least for samples obtained in the morning (34). We therefore used morning samples, adjusted for time of sampling, in our previous GWAMA which identified genetic variants associated with morning plasma cortisol (17). Here, in multivariable regression analyses in both of the two independent studies, a positive association of cortisol with CVD/CHD was only observed when we excluded participants with samples outside of the 0700 to $1100 \mathrm{~h}$ time range. This may be because afternoon cortisol values have less inter-individual variability or because morning cortisol better reflects the reactivity of the hypothalamic-pituitary-adrenal axis or contributes more to the tissue actions of cortisol.

There is also some consistency of findings between cortisol measured in other sample types. The cumulative exposure to cortisol over many weeks or months has been assessed by measuring cortisol in hair, which has also been reported recently to be associated with selfreported history of CVD in a retrospective cohort study, with a comparable magnitude of effect as traditional risk factors (OR: 2.7 for highest cortisol quartile compared to lowest quartile, compared with hypertension (OR: 2.5), abdominal obesity (OR: 2.2) and diabetes mellitus (OR
3.1)) $(35,36)$. Flatter slopes in decline of saliva cortisol across the day in the prospective Whitehall II cohort study among 4047 civil servants were associated with higher risk of cardiovascular deaths (hazard ratio for 1 s.D. reduction in slope steepness $=1.87$; 95\% CI: $1.32-2.64$ ) (37). Also, higher 24-h urinary cortisol levels, as measured in 861 participants who were part of the prospective InCHIANTI study, were associated with higher risk of dying of CVD (highest tertile compared with lowest tertile, hazard ratio $=5.00$; 95\% CI: 2.02-12.37) (38). Of course, we recognise the potential for publication bias in this area so adding genetic evidence to our findings was deemed important.

Mendelian randomization analysis is less likely to be biased by confounding or reverse causation and suggests that the elevated morning plasma cortisol that is associated with CVD/CHD is unlikely to be attributed to, for example, a response of the hypothalamic-pituitaryadrenal axis to chronic inflammation of subclinical cardiovascular disease. There was no strong evidence of an association between the instrumental variable used in the Mendelian randomization analyses and the potential confounders included in the multivariable regression analyses. In addition, estimates from the multivariable Mendelian randomization analyses were consistent with the positive association obtained in the main analyses. The three SNPs comprising the instrumental variable were not in LD $\left(r^{2}<0.1\right)$ with a SNP (rs112635299) associated with CHD in the same locus (29). This suggests it is unlikely that horizontal pleiotropy is biasing the estimate between morning plasma cortisol and CHD observed in our two-sample Mendelian randomization analysis. Further, consistent estimates were obtained using the Wald ratio, inverse variance weighted, likelihood method and weighted median approaches. The genetic variants comprising the instrument in the Mendelian randomization analyses explain $0.5 \%$ variance in morning plasma cortisol. This may cause weak instrument bias which in the two-sample analyses will bias the estimate towards the null.

Greater clarity in understanding the causal pathway between elevated cortisol and CHD will be achieved by firstly, identifying additional variants associated with cortisol to strengthen the instrumental variable used in Mendelian randomization and enable use of additional methods $(39,40,41)$ more adept at handling pleiotropy and secondly, validating the instrumental variable for plasma cortisol in non-European populations justifying the analysis of larger outcome datasets of mixed ancestry leading to greater precision of causal estimates. 
Cortisol acts through intracellular glucocorticoid and mineralocorticoid receptors to modulate fuel metabolism, cardiovascular homeostasis and inflammation. As such there are multiple pathways through which cortisol may be increasing CVD risk. The effects of cortisol on CVD may be mediated by high blood pressure and glucose levels. If this is the case, interventions that target the source of the problem, cortisol, may prevent use of multiple, subsequent interventions (i.e. blood pressure lowering and glucose lowering medications). However, there is also evidence that cortisol is exerting a direct effect on CVD risk, independent of established CVD risk factors. In this case, current treatments will not be effective in reducing CVD risk in individuals with high cortisol levels.

Our stratified analysis suggested there may be a stronger association of morning plasma cortisol with CHD than stroke; although this was not conclusive, it is consistent with the higher risk of CHD, rather than stroke, observed in patients exposed to exogenous glucocorticoids $(6,42)$. The underlying mechanism for this difference is unknown.

The strengths of our studies include the meta-analysis of multiple cohorts, reducing sampling bias and increasing statistical power, and the careful case classification and use of matched controls in the VIP, MONICA and MSP cohort. A further strength is the consistency between different approaches, with the positive prospective association supported by findings from the Mendelian randomization analyses (31).

Limitations include the wide range of sampling times and large number of missing data for sampling time in the VIP, MONICA and MSP cohort, which reduced precision and potentially introduced bias when estimating the association between cortisol and CVD. To address these limitations, we performed multivariable imputation to replace missing sampling times with values imputed from the observed data. When the estimate from the imputed dataset, rather than the observed data, was used to contribute to the meta-analysis then the positive association remained (OR: 1.12, 95\% CI: 1.03-1.22). Given the known importance of sampling time for plasma cortisol, it was not unexpected that there was no association between cortisol and CVD in the unadjusted analysis. The other cardiovascular risk factors that were measured and adjusted for in the studies included in the meta-analysis was not comprehensive so that the adjustments may have been incomplete. The Mendelian randomization within the VIP, MONICA and MSP cohort, which aimed to establish three-way associations between genotype, plasma cortisol and CVD, was under-powered.
Improving our understanding of the underlying mechanisms of CVD, as well as identifying biomarkers, is of public health importance as it can lead to the development of novel pharmaceutical treatments and guide targeted interventions for those most at risk. The current data suggest that strategies targeted at lowering cortisol action may reduce risk of CVD. Reducing cortisol action safely is challenging, given that the anti-inflammatory properties of cortisol are essential to survival during, for example, severe infection. However, approaches which target tissue-specific cortisol action, such as inhibition of the cortisol regenerating enzyme 11ßHSD1, have shown modest improvements in glycaemic control, lipid profile and blood pressure in phase II clinical trials for type 2 diabetes and arguably should be evaluated for their effects on CVD (43).

\section{Supplementary data}

This is linked to the online version of the paper at https://doi.org/10.1530/ EJE-19-0161.

\section{Declaration of interest}

B R W reports grants from British Heart Foundation, Scottish Government Chief Scientist Office and Wellcome Trust. S S reports grants from Swedish Heart and Lung Foundation, grants from County Councils in Norr- and Västerbotten. B R W is an inventor on patents owned by the University of Edinburgh for $11 \beta$-HSD1 inhibitors, which have been licenced to Actinogen Medical Ltd, a company for which BRW undertakes paid consultancy. The other authors have nothing to disclose.

\section{Funding}

The authors are grateful for research funding from the British Heart Foundation (to A A C, R M R, B R W), Scottish Government Chief Scientist Office and Wellcome Trust (to B R W). The VIP, MONICA and MSP studies were supported by the county councils in Norr- and Västerbotten, Sweden, and Stefan Söderberg was supported by the Swedish Heart and Lung Foundation and the County Council of Västerbotten (ALF). G D S, N J T and $D A L$ work in a unit that receives support from the University of Bristol and UK Medical Research Council (MR_UU_12013/1, MR_UU_12013/5).

\section{Acknowledgements}

The authors wish to acknowledge the Västerbotten Intervention Project (VIP), the Northern Sweden MONICA project and the County Council of Västerbotten and the participants, nursing and administrative staff supporting all of the studies. They are grateful to supporting staff and the EBF-unit (Åsa Ågren and staff) at Umeå University. They thank Jill Harrison and Audrey Duncan for technical support.

\section{References}

1 Shepherd J, Cobbe SM, Ford I, Isles CG, Lorimer AR, Macfarlane PW, McKillop JH \& Packard CJ. Prevention of coronary heart disease with pravastatin in men with hypercholesterolemia. West of Scotland Coronary Prevention Study Group. New England Journal 
of Medicine 1995333 1301-1307. (https://doi.org/10.1056/ NEJM199511163332001)

2 Bangalore S, Steg G, Deedwania P, Crowley K, Eagle KA, Goto S, Ohman EM, Cannon CP, Smith SC, Zeymer U et al. $\beta$-Blocker use and clinical outcomes in stable outpatients with and without coronary artery disease. JAMA 2012308 1340-1349. (https://doi.org/10.1001/ jama.2012.12559)

3 Alwan A. Global Status Report on Noncommunicable Diseases 2010. World Health Organization, 2011.

4 Etxabe J \& Vazquez JA. Morbidity and mortality in Cushing's disease: an epidemiological approach. Clinical Endocrinology 199440 479-484. (https://doi.org/10.1111/j.1365-2265.1994.tb02486.x)

5 Faggiano A, Pivonello R, Spiezia S, De Martino MC, Filippella M, Di Somma C, Lombardi G \& Colao A. Cardiovascular risk factors and common carotid artery caliber and stiffness in patients with Cushing's disease during active disease and 1 year after disease remission. Journal of Clinical Endocrinology and Metabolism $2003 \mathbf{8 8}$ 2527-2533. (https://doi.org/10.1210/jc.2002-021558)

6 Wei L, MacDonald TM \& Walker BR. Taking glucocorticoids by prescription is associated with subsequent cardiovascular disease. Annals of Internal Medicine 2004141 764-770. (https://doi. org/10.7326/0003-4819-141-10-200411160-00007)

7 Walker BR. Abnormal glucocorticoid activity in subjects with risk factors for cardiovascular disease. Endocrine Research 199622 701-708. (https://doi.org/10.1080/07435809609043765)

8 Filipovsky J, Ducimetiere P, Eschwege E, Richard JL, Rosselin G \& Claude JR. The relationship of blood pressure with glucose, insulin, heart rate, free fatty acids and plasma cortisol levels according to degree of obesity in middle-aged men. Journal of Hypertension 1996 14 229-235. (https://doi.org/10.1097/00004872-199602000-00012)

9 Fraser R, Ingram MC, Anderson NH, Morrison C, Davies E \& Connell JM. Cortisol effects on body mass, blood pressure, and cholesterol in the general population. Hypertension $1999331364-$ 1368. (https://doi.org/10.1161/01.HYP.33.6.1364)

10 Reynolds RM, Walker BR, Syddall HE, Andrew R, Wood PJ \& Phillips DI. Is there a gender difference in the associations of birthweight and adult hypothalamic-pituitary-adrenal axis activity? European Journal of Endocrinology 2005152 249-253. (https://doi. org/10.1530/eje.1.01846)

11 Hamer M, O'Donnell K, Lahiri A \& Steptoe A. Salivary cortisol responses to mental stress are associated with coronary artery calcification in healthy men and women. European Heart Journal 2010 31 424-429. (https://doi.org/10.1093/eurheartj/ehp386)

12 Reynolds RM, Ilyas B, Price JF, Fowkes FGR, Newby DE, Webb DJ $\&$ Walker BR. Circulating plasma cortisol concentrations are not associated with coronary artery disease or peripheral vascular disease. QJM 2009102 469-475. (https://doi.org/10.1093/qjmed/hcp057)

13 Davey Smith GD, Ben-Shlomo Y, Beswick A, Yarnell J, Lightman S \& Elwood P. Cortisol, testosterone, and coronary heart disease prospective evidence from the Caerphilly Study. Circulation 2005112 332-340. (https://doi.org/10.1161/CIRCULATIONAHA.104.489088)

14 Rod NH, Kristensen TS, Diderichsen F, Prescott E, Jensen GB \& Hansen AM. Cortisol, estrogens and risk of ischaemic heart disease, cancer and all-cause mortality in postmenopausal women: a prospective cohort study. International Journal of Epidemiology 2010 39 530-538. (https://doi.org/10.1093/ije/dyp354)

15 Phillips AC, Carroll D, Gale CR, Lord JM, Arlt W \& Batty GD. Cortisol, DHEA sulphate, their ratio, and all-cause and cause-specific mortality in the Vietnam Experience Study. European Journal of Endocrinology 2010163 285-292. (https://doi.org/10.1530/EJE-100299)

16 Davey Smith GD \& Ebrahim S. 'Mendelian randomization': can genetic epidemiology contribute to understanding environmental determinants of disease? International Journal of Epidemiology 200332 1-22. (https://doi.org/10.1093/ije/dyg070)
17 Bolton JL, Hayward C, Direk N, Lewis JG, Hammond GL, Hill LA, Anderson A, Huffman J, Wilson JF, Campbell H et al. Genome wide association identifies common variants at the SERPINA6/SERPINA1 locus influencing plasma cortisol and corticosteroid binding globulin. PLoS Genetics 201410 e1004474. (https://doi.org/10.1371/ journal.pgen.1004474)

18 Norberg M, Wall S, Boman K \& Weinehall L. The Västerbotten Intervention Programme: Background, design and implications. Global Health Action 20103 4643. (https://doi.org/10.3402/gha. v3i0.4643)

19 Stegmayr B, Lundberg V \& Asplund K. The events registration and survey procedures in the Northern Sweden MONICA Project. Scandinavian Journal of Public Health: Supplement 200361 9-17. (https://doi.org/10.1080/14034950310001441)

20 Tunstall-Pedoe H, Kuulasmaa K, Amouyel P, Arveiler D, Rajakangas AM \& Pajak A. Myocardial infarction and coronary deaths in the World Health Organization MONICA Project. Registration procedures, event rates, and case-fatality rates in 38 populations from 21 countries in four continents. Circulation 1994 90 583-612. (https://doi.org/10.1161/01.CIR.90.1.583)

21 Whitworth JA, Stewart PM, Burt D, Atherden SM \& Edwards CR. The kidney is the major site of cortisone production in man. Clinical Endocrinology 198931 355-361. (https://doi. org/10.1111/j.1365-2265.1989.tb01259.x)

22 Lawlor DA, Bedford C, Taylor M \& Ebrahim S. Geographical variation in cardiovascular disease, risk factors, and their control in older women: British Women's Heart and Health Study. Journal of Epidemiology and Community Health 200357 134-140. (https://doi. org/10.1136/jech.57.2.134)

23 Lawlor DA, Davey Smith G, Ebrahim S, Thompson C \& Sattar N. Plasma adiponectin levels are associated with insulin resistance, but do not predict future risk of coronary heart disease in women. Journal of Clinical Endocrinology and Metabolism 200590 5677-5683. (https:// doi.org/10.1210/jc.2005-0825)

24 Pearce N. Analysis of matched case-control studies. BMJ 2016352 i969. (https://doi.org/10.1136/bmj.i969)

25 White IR, Royston P \& Wood AM. Multiple imputation using chained equations: issues and guidance for practice. Statistics in Medicine 201130 377-399. (https://doi.org/10.1002/sim.4067)

26 StataCorp. Stata Statistical Software: Release 15, 15th ed. College Station, TX: StataCorp LLC, 2017.

27 Higgins JP \& Thompson SG. Quantifying heterogeneity in a meta-analysis. Statistics in Medicine 200221 1539-1558. (https://doi. org/10.1002/sim.1186)

28 Burgess S, Scott RA, Timpson NJ, Davey Smith G \& Thompson SG \& EPIC-InterAct Consortium. Using published data in Mendelian randomization: a blueprint for efficient identification of causal risk factors. European Journal of Epidemiology 201530 543-552. (https:// doi.org/10.1007/s10654-015-0011-z)

29 van der Harst P \& Verweij N. Identification of 64 novel genetic loci provides an expanded view on the genetic architecture of coronary artery disease. Circulation Research 2018122 433-443. (https://doi. org/10.1161/CIRCRESAHA.117.312086)

30 Wald A. The fitting of straight lines if both variables are subject to error. Annals of Mathematical Statistics 194011 284-300. (https://doi. org/10.1214/aoms/1177731868)

31 Lawlor DA, Tilling K \& Davey Smith G. Triangulation in aetiological epidemiology. International Journal of Epidemiology 201645 18661886. (https://doi.org/10.1093/ije/dyw314)

32 Walker BR. Glucocorticoids and cardiovascular disease. European Journal of Endocrinology 2007157 545-559. (https://doi.org/10.1530/ EJE-07-0455)

33 Reynolds RM, Walker BR, Syddall HE, Andrew R, Wood PJ, Whorwood CB \& Phillips DIW. Altered control of cortisol secretion in adult men with low birth weight and cardiovascular risk factors 
1. Journal of Clinical Endocrinology and Metabolism 200186 245-250. (https://doi.org/10.1210/jcem.86.1.7145)

34 Reynolds RM, Fischbacher C, Bhopal R, Byrne CD, White M, Unwin N \& Walker BR. Differences in cortisol concentrations in South Asian and European men living in the United Kingdom. Clinical Endocrinology 200664 530-534. (https://doi.org/10.1111/ j.1365-2265.2006.02504.x)

35 Manenschijn L, Schaap L, Van Schoor NM, Van der Pas S, Peeters GMEE, Lips P, Koper JW \& Van Rossum EFC. High long-term cortisol levels, measured in scalp hair, are associated with a history of cardiovascular disease. Journal of Clinical Endocrinology and Metabolism 201398 2078-2083. (https://doi.org/10.1210/jc.2012-3663)

36 Yusuf S, Hawken S, Ônpuu S, Dans T, Avezum A, Lanas F, McQueen M, Budaj A, Pais P, Varigos J et al. Effect of potentially modifiable risk factors associated with myocardial infarction in 52 countries (the INTERHEART study): case-control study. Lancet 2004 364 937-952. (https://doi.org/10.1016/S0140-6736(04)17018-9)

37 Kumari M, Shipley M, Stafford M \& Kivimaki M. Association of diurnal patterns in salivary cortisol with all-cause and cardiovascular mortality: findings from the Whitehall II study. Journal of Clinical Endocrinology and Metabolism 201196 1478-1485. (https://doi. org/10.1210/jc.2010-2137)

38 Vogelzangs N, Beekman AT, Milaneschi Y, Bandinelli S, Ferrucci L $\&$ Penninx BW. Urinary cortisol and six-year risk of all-cause and cardiovascular mortality. Journal of Clinical Endocrinology and Metabolism 201095 4959-4964. (https://doi.org/10.1210/jc.20100192)

39 Bowden J, Davey Smith G, Haycock PC \& Burgess S. Consistent estimation in Mendelian randomization with some invalid instruments using a weighted median estimator. Genetic Epidemiology 201640 304-314. (https://doi.org/10.1002/ gepi.21965)

40 Holmes MV, Ala-Korpela M \& Davey Smith G. Mendelian randomization in cardiometabolic disease: challenges in evaluating causality. Nature Reviews: Cardiology 201714 577-590. (https://doi. org/10.1038/nrcardio.2017.78)

41 Bowden J, Davey Smith G \& Burgess S. Mendelian randomization with invalid instruments: effect estimation and bias detection through Egger regression. International Journal of Epidemiology 2015 44 512-525. (https://doi.org/10.1093/ije/dyv080)

42 Souverein PC, Berard A, Van Staa TP, Cooper C, Egberts AC, Leufkens HG \& Walker BR. Use of oral glucocorticoids and risk of cardiovascular and cerebrovascular disease in a population based case-control study. Heart 200490 859-865. (https://doi.org/10.1136/ hrt.2003.020180)

43 Anderson A \& Walker BR. 11 $\beta$-HSD1 inhibitors for the treatment of type 2 diabetes and cardiovascular disease. Drugs 2013731385 1393. (https://doi.org/10.1007/s40265-013-0112-5)

Received 5 March 2019

Revised version received 12 June 2019

Accepted 18 July 2019 